\title{
TIÑA VULVAR: ANÁLISIS HISTOPATOLÓGICO DE UN CASO
}

\section{Tinea of the vullva: a histopathological case anallysis}

Iván L. Mojica, M.D. *, Viviana Arias, M.D.**, Gerzaín Rodríguez, M.D.***

Recibido: agosto 19/09 - Aceptado: mayo 24/10

\section{RESUMEN}

Introducción: la tiña vulvar es una entidad de difícil diagnóstico clínico que puede también pasar desapercibida en la biopsia.

Objetivo: presentar el caso de una mujer con esta entidad, diagnosticado por la biopsia, y hacer comentarios relevantes sobre el tema con énfasis en el diagnóstico histopatológico.

Metodología: se trata de una paciente sometida a biopsia por prurito vulvar persistente. No se anotan otros datos de historia clínica, incluida la edad. En la biopsia se observó una dermatitis psoriasiforme liquenificada, con paraqueratosis y grupos de neutrófilos en la capa córnea y con tapones córneos infundibulares; en la dermis superficial se apreciaban papilas profundas con algunos linfocitos y fibrosis vertical. Se sospechó el diagnóstico de psoriasis pero la coloración de PAS (coloración de ácido peryódico de Schiff), solicitada por la presencia de neutrófilos intracórneos, demostró abundantes hifas en un infundíbulo confirmándose el diagnóstico de tiña vulvar.

Conclusiones: la entidad demostrada es rara y tiene como uno de sus patrones histopatológicos la presencia

\footnotetext{
* Residente III de Patología Anatómica y Clínica, Facultad de Medicina, Universidad Nacional. Bogotá (Colombia). Correo electrónico: ilmojicaf@unal.edu.co

** Profesora Asistente de Patología, Facultad de Medicina, Universidad Nacional. Bogotá (Colombia).

*** Profesor Titular y Maestro Universitario (Jubilado), Facultad de Medicina, Universidad Nacional. Bogotá (Colombia). Catedrático, Facultad de Medicina, Universidad de la Sabana. Chía (Cundinamarca) Departamento de Patología, Universidad Nacional de Colombia. Bogotá (Colombia). Correo electrónico: gerzain_rodriguez@yahoo.com
}

de una dermatitis psoriasiforme y liquenificada. El hallazgo de neutrófilos intracórneos siempre debe hacer sospechar la presencia de una tiña. La coloración de PAS es una manera fácil de confirmar el diagnóstico. En la literatura revisada se constata que la tiña vulvar es una extensión de la tiña cruris, producida con mayor frecuencia por Trichophyton rubrum. Los diagnósticos diferenciales incluyen candidiasis, psoriasis y dermatitis de contacto.

Palabras clave: vulva/lesiones, vulva/patología, vulvitis/patología, vulvitis/microbiología, vulvitis/diagnóstico, tiña/etiología, tiña/patología, tiña/microbiología, prurito/etiología, dermatomicosis/patología.

\section{SUMMARY}

Introduction: tinea of the vulva is a difficult clinical entity which may well go unnoticed in a biopsy.

Objective: presenting the case of a woman suffering this disease, diagnosed by biopsy, and making some comments emphasising the value of histopathological diagnosis.

Methodology: a vulvar biopsy was taken as the patient had complained of persistent pruritis. No clinical history was obtained. The biopsy revealed lichenified psoriasiform dermatitis, parakeratosis and groups of neutrophils in the horny layer and infundibulum; deep dermal papillae, lymphocytes and vertical fibrosis were visible in the dermis. Although such findings are also seen in psoriasis, PAS staining was done due to the presence of neutrophils, showing abundant infundibular hyphae, thereby confirming the diagnosis of tinea of the vulva. 
Conclusions: this rare entity has a lichenified psoriasiform histological pattern. The finding of neutrophils in the horny layer must always lead to suspecting the presence of tinea. PAS staining is an easy way to confirm such diagnosis. Reviewing the literature revealed that tinea of the vulva is an extension of tinea cruris, most frequently caused by Trichophyton rubrum. Differential diagnosis includes candidiasis, psoriasis, and contact dermatitis.

Key words: dermatomycoses/epidemiology, dermatomycoses/microbiology, vulvar disease, pruritus/aetiology, vulvitis/aetiology, vulvitis/ pathology, tinea/microbiology, tinea/pathology.

\section{INTRODUCCIÓN}

El término "tinea" o "tiña" se utiliza para denominar a las enfermedades micóticas superficiales causadas por hongos que tienen la capacidad de invadir la capa córnea de la piel, el peloy las uñas, ${ }^{1}$ denominados dermatofitos, incluidos en los géneros Trichophyton, Epydermophyton y Microsporum. Los hongos de los géneros Malassezia y Candida también pueden invadir la capa córnea de la piel y las mucosas pero no son auténticos dermatofitos. ${ }^{2}$

Las tiñas son las enfermedades dermatológicas más comunes en el mundo con alrededor del 5\% de los motivos de consulta ambulatoria; ${ }^{1,3}$ sin embargo, la tiña vulvar es una enfermedad rara considerada una extensión de la tiña cruris. ${ }^{4}$ La tiña cruris es más frecuente en hombres que en mujeres y puede comprometer cualquier individuo sin importar la edad;, ${ }^{1,2}$ el pico más alto ocurre en los adultos jóvenes, ${ }^{1}$ principalmente los deportistas, militares y nadadores que están en mayor riesgo de desarrollar la enfermedad por las características de humedad de la zona inguinocrural y genital facilitadas por la sudoración y el tipo de ropa utilizada. ${ }^{1,2}$

El objetivo de este artículo es presentar el caso de una mujer con prurito vulvar cuyo diagnóstico definitivo por biopsia fue tiña vulvary hacer comentarios relevantes sobre el tema con énfasis en el diagnóstico histopatológico.

\section{METODOLOGÍA}

Se trata de una paciente a quién se le realizó una biopsia de piel de la vulva por presentar prurito. No contamos con otros datos como la edad o descripción clínica de las lesiones. La biopsia es un fragmento de piel de $4 \times 3 \times 3 \mathrm{~mm}$. La epidermis presenta acantosis psoriasiforme, espongiosis discreta, paraqueratosis focal y exocitosis de neutrófilos que forman pequeñas colecciones subcórneas. Se observa también un tapón córneo infundibular paraqueratósico con neutrófilos en su interior. En la dermis las papilas son profundas y se ve discreto infiltrado linfocitario superficial (figuras 1-4). Se practicó coloración de ácido peryódico de Schiff (PAS) que demostró abundantes hifas en el estrato córneo y en un infundíbulo (figura 5). Se hizo el diagnóstico de tiña vulvar.

Figura 1. Epidermis con acantosis psoriasiforme de las crestas interpapilares, un tapón córneo infundibular y delgada capa córnea con paraqueratosis. La dermis presenta edema, papilas profundas e infiltrado inflamatorio moderado superficial. H\&E $4 \mathrm{X}$

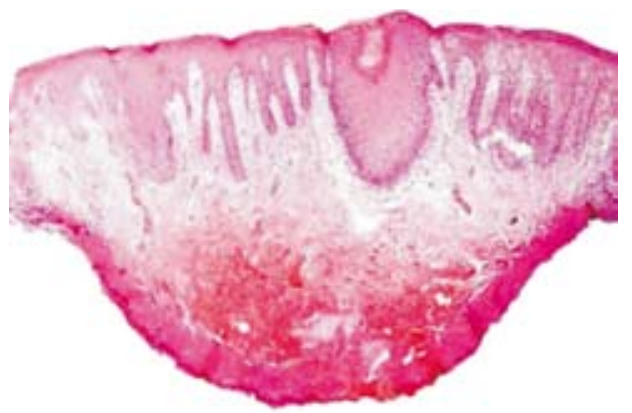

Figura 2. A mayor aumento se destaca el tapón córneo infundibular paraqueratósico, la acantosis psoriasiforme y la delgada capa córnea paraqueratósica. Hay algunos neutrófilos en el borde infundibular derecho, que se amplían en la figura 3. En la dermis, las papilas son profundas con fibrosis vertical, vasos congestivos e infiltrado linfocitario. H\&E 10X.

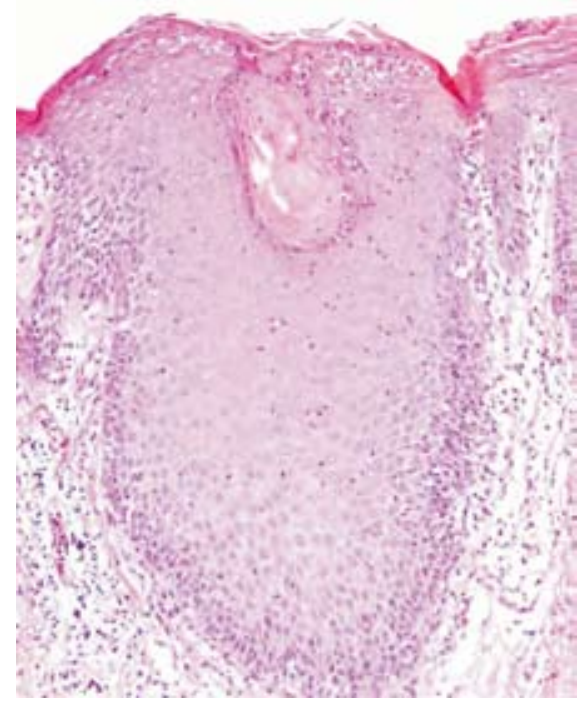


Figuras 3 y 4. La figura 3 es una ampliación de la figura 2. Tanto en la figura 3 como en la 4 se ven abundantes neutrófilos que forman colecciones subcórneas e intramalpighianas. Este hallazgo es común a la psoriasis y a las tiñas. H\&E 40X
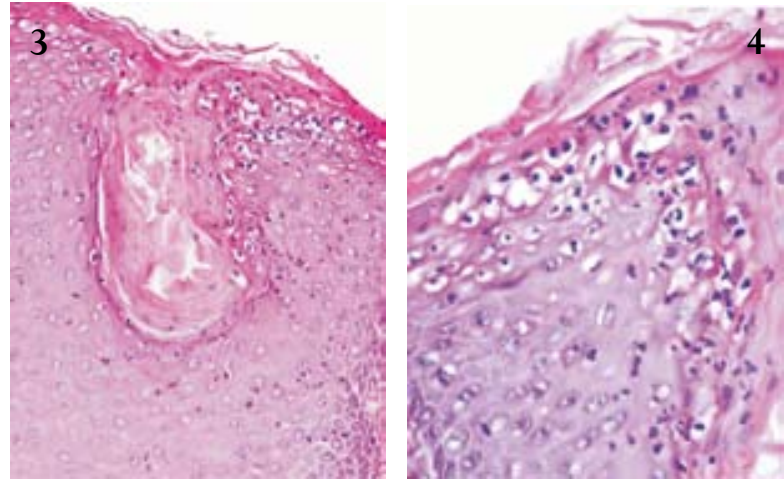

Figura 5. La coloración de PAS demuestra abundantes hifas en el infundíbulo. 40x.

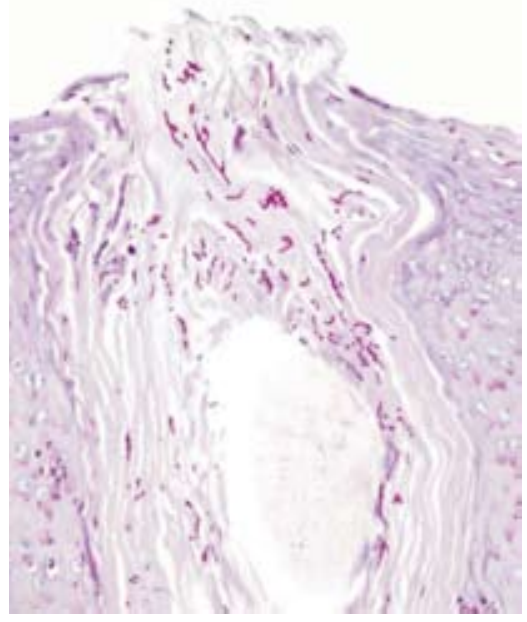

\section{DISCUSIÓN}

La tiña vulvar es una enfermedad rara y se considera una extensión de la tiña cruris, encontrándose esta última en cerca del 5\% de las pacientes que consultan por dermatosis en los genitales femeninos externos. ${ }^{1}$ Trichophyton rubrum es el dermatofito más frecuentemente aislado en la tiña vulvar, en el 32 al 60\% de los casos, seguido por Trichophyton tonsurans y Trichophyton mentagrophytes. ${ }^{1}$ Las pacientes consultan por presentar dolor tipo ardor, sensación de calor vulvar, dispareunia y disuria. En el examen físico se pueden encontrar múltiples placas eritematosas y edematosas, de centro más claro y de borde levantado, asociadas con lesiones satélites que comprometen los labios mayores, la región inguinal y la perianal. Además, puede observarse un importante engrosamiento epitelial por liquenificación ocasionada por el prurito. Ocasionalmente se pueden presentar procesos supurativos concomitantes, probablemente secundarios a escoriaciones por el rascado que se impetiginizan y que orientan hacia una etiología bacteriana sin respuesta clínica después de recibir tratamiento antibiótico. ${ }^{4,5}$

Histológicamente, en la tiña vulvar se puede encontrar dermatitis espongiótica, vesicular o psoriasiforme, con moderado a severo infiltrado inflamatorio perivascular en la dermis papilar compuesto principalmente por linfocitos y algunos histiocitos. La presencia de neutrófilos que permean la epidermis llegando hasta el estrato córneo es clave para el diagnóstico (figuras 1-4). También es común la hiperqueratosis y la paraqueratosis. Cuando el proceso inflamatorio es crónico pueden observarse células plasmáticas. ${ }^{4,6,7}$ En el caso presentado, la imagen histológica muestra una dermatitis psoriasiforme que podría corresponder a psoriasis, tiña o dermatitis de contacto, liquenificada. La presencia de neutrófilos obliga a realizar la coloración de PAS que demuestra claramente las hifas intracórneas e infundibulares (figura 5). Las hifas se pueden evidenciar en la coloración de hematoxilina- eosina como estructuras elongadas birrefringentes en el estrato córneo, con el objetivo de 40X y bajando el condensador.

El agente etiológico que siempre debe hacer parte del diagnóstico diferencial con estos hallazgos es el Candida sp., hongo responsable de cerca del $10 \%$ de las dermatosis vulvovaginales en mujeres inmunocompetentes. ${ }^{6}$ Clínicamente, la diferencia entre dermatofitosis y candidiasis es muy difícil. Histológicamente, en la candidiasis el infiltrado inflamatorio puede ser más severo y los neutrófilos pueden formar micropústulas en el estrato córneo, con marcado edema en la dermis papilar subyacente. ${ }^{6,7}$ La identificación de pseudohifas y blastoconidias ovoides sugiere la presencia de Candida sp. Las pseudohifas son 
estructuras elongadas y septadas que miden hasta 4 micras de diámetro y presentan una angulación hasta de $90^{\circ}$ en el punto de ramificación, en donde presenta un aspecto constreñido del septo. ${ }^{7}$ Tienden a orientarse verticalmente en la capa córnea lo cual es una clave para su identificación. Ocasionalmente, la observación de las típicas “albóndigas y espaguetis” en el estrato córneo, sin mayor inflamación dérmica, sugiere la participación de Malassezia sp. como agente etiológico, un hongo responsable de la tiña versicolor. ${ }^{6}$ En todo caso, el cultivo identifica el agente causal y ayuda a seleccionar el tratamiento.

El diagnóstico diferencial de la tiña vulvar incluye entidades no infecciosas como la psoriasis, la cual presenta acantosis con elongación regular de las crestas interpapilares, hipogranulosis focal y paraqueratosis en montículos, con espongiosis leve; la exocitosis de neutrófilos, que llega a formar colecciones en el estrato córneo (microabscesos de Munro), ocurre tanto en la psoriasis como en las tiñas y es una indicación para realizar la coloración de PAS, que revela con claridad los hongos (figura 5) en la capa córnea. La ausencia de hongos sugiere el diagnóstico de psoriasis.

En la dermatitis de contacto predominan la espongiosis, la acantosis con hipergranulosis y la paraqueratosis con vesiculillas córneas; en la dermis el infiltrado inflamatorio linfocitario superficial puede acompañarse de eosinófilos ${ }^{7}$ y si la lesión es crónica y pruriginosa, hay cambios de liquenificación que consisten en hiperqueratosis compacta, hipergranulosis, acantosis psoriasiforme y en la dermis, papilas profundas con haces colágenos orientados verticalmente, una forma histológica de identificar el prurito. El infiltrado inflamatorio es linfocitario, escaso.

\section{CONCLUSIONES}

La tiña vulvar es una entidad rara que se considera una extensión de la tiña cruris; histológicamente debe sospecharse cuando se observa vulvitis con neutrófilos intracórneos, una indicación para practicar la coloración de PAS, que revela las hifas intracórneas y en los infundíbulos.

\section{REFERENCIAS}

1. Hernández A, Carvajal P. Dermatofitosis por Trichophyton rubrum. Experiencia de 10 años (19962005) en un servicio de dermatología de un hospital general de la Ciudad de México. Rev Iberoam Micol 2007;24:122-4.

2. Walker TS. Hongos que ocasionan micosis superficiales, cutáneas y subcutáneas. En: Walker TS. Microbiología Clínica. $1^{\text {a }}$ ed. México: McGraw-Hill Interamericana; 1998. p. 317-20.

3. Foster K, Ghamoum MA, Elewski BE. Epidemiologic surveillance of cutaneous fungal infection in the United States from 1999 to 2002. J Am Acad Dermatol 2004;50:748-52.

4. Barile F, Filotico R, Cassano N, Vena GA. Pubic and vulvar inflammatory tinea due to Trichophyton mentagrophytes. Int J Dermatol 2006;45:1369-70.

5. Singh N, Thappa DM , Thappa DM, Jaisankar TJ, Habeebullah S. Pattern of non-venereal dermatoses of female external genitalia in South India. Dermatol Online J 2008;14:1.

6. Hammock L, Barrett T. Inflammatory dermatoses of the vulva. J Cutan Pathol 2005;32:604-11.

7. Hinshaw M, Longley BJ. Fungal diseases, In: Elder D, Editor. Lever's Histopathology of the skin. Tenth edition. Philadelphia: Lippincott Williams \& Wilkins; 2009. p. 591. 